Recursive low rank Hankel approximation and model reduction.

Chahlaoui, Younes and Van Dooren, Paul

2003

MIMS EPrint: 2008.19

Manchester Institute for Mathematical Sciences

School of Mathematics

The University of Manchester

\footnotetext{
Reports available from: http://eprints.maths.manchester.ac.uk/

And by contacting: The MIMS Secretary

School of Mathematics

The University of Manchester

Manchester, M13 9PL, UK
} 


\title{
RECURSIVE LOW RANK HANKEL APPROXIMATION AND MODEL REDUCTION
}

\author{
Y. Chahlaoui*, P. Van Dooren ${ }^{\dagger}$ \\ CESAME, Université Catholique de Louvain \\ Bat.Euler, 4 Av.G.Lemaitre, B-1348 Louvain-La-Neuve, Belgium. \\ fax: +3210472180 \\ e-mail: *chahlaoui@csam.ucl.ac.be †vdooren@csam.ucl.ac.be \\ http://www.csam.ucl.ac.be/ $\{$ chahloui, vdooren $\}$
}

Keywords: Model reduction, time-varying systems, Hankel maps, Gramians, Low-rank approximation.

\begin{abstract}
In this note we present a new updating technique to estimate a low rank approximation of the Hankel map of a time-varying system. We obtain error estimates of our approximation and also explain how to use this for model reduction of time-varying as well as time invariant systems.
\end{abstract}

\section{Introduction}

In the last twenty years, a variety of model reduction techniques have been developed to produce systematically simpler models of complex systems which is essentially a mathematical model describing a real world physical process. One wants that the mathematical model reflects the physics of the real world process, and satisfying this rigorously often requires models of very high complexity. For such cases it is essential to design models of reduced complexity.

A popular model reduction technique is balanced truncation (BT) [7] [3] for which an a priori error bound on the truncated model can be obtained [4]. This approach "balances out" the states so that each state is as controllable as it is observable; one then just truncates the "weak" states to produce a good

*This paper presents research supported by the Belgian Programme on Inter-university Poles of Attraction, initiated by the Belgian State, Prime Minister's Office for Science, Technology and Culture. This work was also supported by the National Science Foundation under Grant No. CCR20003050. The work of the first author has been partially carried out within the framework of a collaboration agreement between CESAME (Université Catholique de Louvain, Belgium) and LINMA of the Faculty of Sciences (Université Chouaib Doukkali, Morocco), funded by the Secretary of the State for Development Cooperation and by the CIUF (Conseil Interuniversitaire de la Communauté Française, Belgium). reduced order model. In [6] and [9] a generalization to time-varying systems is presented, but without any algorithmic details. Recently, an a priori error bound for time-varying systems that is similar to the timeinvariant case was obtained in [8].

The present paper focuses on the case were the original system is large and sparse. This is typically the case if it originates from a spatial discretization of partial differential equations (see e.g. [11]). For such systems it is important to exploit this sparsity for computational efficiency. Iterative methods are very suitable for this and are often easy to parallelize as well. Direct methods like balanced truncation, on the other hand, ignore sparsity in the system and are not very attractive for parallelization : if we denote the order of the system by $N$, the complexity of these methods is roughly $\Theta\left(N^{3}\right)$ flops and they require about $\Theta\left(N^{2}\right)$ words of memory, which is too expensive computationally to use on large problems. In the sequel we describe a novel approach for iterative projection. It uses the Hankel operators which are mainly related to controllability and observability Gramians. The computational cost for constructing those is just about $\Theta(N)$ flops at each iteration, and we obtain a similar results of those obtained via BT. All our results are presented for linear discrete-time systems, but they extend to linear continuous-time systems as well.

\section{Time-varying systems}

Linear discrete time-varying systems are described by systems of difference equations:

$$
x_{k+1}=A_{k} x_{k}+B_{k} u_{k}, \quad y_{k}=C_{k} x_{k}
$$

with input $u_{k} \in \mathbb{R}^{m}$, state $x_{k} \in \mathbb{R}^{N}$ and output $y_{k} \in \mathbb{R}^{p}$, and $m, p<<N$. For infinite behaviour problems, the input sequence is assumed to 
be square-summable, i.e $u_{k} \in l_{2}^{m} 1$, and we assume that $\left\{A_{k}\right\}_{0}^{\infty},\left\{B_{k}\right\}_{0}^{\infty}$, and $\left\{C_{k}\right\}_{0}^{\infty}$ are bounded sequences $^{2}$ of matrices with appropriate dimensions. Using the recurrence (1) over several time steps, one obtains the state at step $k$ in function of past inputs over the interval $\left[k_{i}, k\right]$ :

$$
x_{k}=\Phi\left(k, k_{i}\right) x_{k_{i}}+\sum_{i=k_{i}}^{k-1} \Phi(k, i+1) B_{i} u_{i},
$$

where $\Phi\left(k, k_{i}\right) \doteq A_{k-1} \ldots A_{k_{i}}$ is the discrete transition matrix over time period $\left[k_{i}, k\right]$. We will assume the time-varying system to be asymptotically stable, which means that $\forall k \geq k_{i}$

$$
\left\|\Phi\left(k, k_{i}\right)\right\|_{2} \leq m \cdot a^{\left(k-k_{i}\right)} \text {, with } m \geq 1,0 \leq a<1 .
$$

Under such conditions one can define the Gramians over intervals $\left[k_{i}, k-1\right]$ and $\left[k, k_{f}\right]$ as follows :

$$
\begin{gathered}
\mathcal{G}_{c}(k)=\sum_{i=k_{i}}^{k-1} \Phi(k, i+1) B_{i} B_{i}^{T} \Phi^{T}(k, i+1), \\
\mathcal{G}_{o}(k)=\sum_{i=k}^{k_{f}} \Phi^{T}(i, k) C_{i}^{T} C_{i} \Phi(i, k) .
\end{gathered}
$$

(Notice that the asymptotic stability is needed only when $k_{i}=-\infty$ or $k_{f}=+\infty$.)

These Gramians can also be obtained from the Stein recurrence formulas:

$$
\mathcal{G}_{c}(k+1)=A_{k} \mathcal{G}_{c}(k) A_{k}^{T}+B_{k} B_{k}^{T},
$$

and

$$
\mathcal{G}_{o}(k)=A_{k}^{T} \mathcal{G}_{o}(k+1) A_{k}+C_{k}^{T} C_{k},
$$

with respective initial conditions

$$
\mathcal{G}_{c}\left(k_{i}\right)=\mathbf{0}, \quad \mathcal{G}_{o}\left(k_{f}+1\right)=\mathbf{0} .
$$

We point out that both recurrences (2) and (3) evolve differently with time. The equation (2) goes "forward" in time, while the equation (3) goes "backward" in time.

\footnotetext{
${ }^{1}$ this is a Hilbert space with inner product $\langle x, y\rangle_{l_{2}^{m}} \doteq$ $\sum_{-\infty}^{\infty} x_{k}^{T} y_{k}$

${ }^{2}$ A sequence of matrices $\left\{M_{k}\right\}$ is said to be bounded if there exists a constant $M \in \mathbb{R}$ such that $\left\|M_{k}\right\|_{2} \leq M, \forall k \in \mathbb{Z}$.
}

\section{Recursive low-rank Hankel method}

Let us consider a time window $\left[k_{i}, k_{f}\right]=[k-\tau, k+$ $\tau-1]$ of width $2 \tau$ and centered around $[k-1, k]$. If we restrict the inputs to be non-zero only in the interval $\left[k_{i}, k-1\right]$ (i.e. the "past"), then the outputs in the interval $\left[k, k_{f}\right]$ (i.e. the "future") are given by the convolution with a "Hankel map". Indeed, the state-to-outputs and inputs-to-state maps on the finite window $\left[k_{i}, k_{f}\right]$ are given by $\left(k_{i}<k \leq k_{f}\right)$ [12]:

$$
Y=\mathcal{O}\left(k, k_{f}\right) \underbrace{\mathcal{C}\left(k, k_{i}\right) U}_{x(k)},
$$

where $Y \doteq\left[\begin{array}{lll}y_{k}^{T} & \ldots & y_{k_{f}}^{T}\end{array}\right]^{T}, U \doteq\left[\begin{array}{lll}u_{k-1}^{T} & \ldots & u_{k_{i}}^{T}\end{array}\right]^{T}$, $\mathcal{O}\left(k, k_{f}\right) \doteq\left[\begin{array}{lllll}C_{k}^{T} & A_{k}^{T} C_{k+1}^{T} & \ldots & \Phi\left(k_{f}, k\right)^{T} C_{k_{f}}^{T}\end{array}\right]$, and $\mathcal{C}\left(k, k_{i}\right) \doteq\left[\begin{array}{llll}B_{k-1} & A_{k-1} B_{k-2} & \ldots & \Phi\left(k, k_{i}+1\right) B_{k_{i}}\end{array}\right]$, where $\mathcal{O}_{k} \doteq \mathcal{O}\left(k, k_{f}\right)$ and $\mathcal{C}_{k} \doteq \mathcal{C}\left(k, k_{i}\right)$ are respectively the observability and the reachability matrices at instant $k$ related to the finite windows $\left[k, k_{f}\right]$ and $\left[k_{i}, k-1\right]$. Since $x(k) \in \mathbb{R}^{N}$, the finite dimensional "Hankel" map $\mathcal{H}\left(k, k_{i}, k_{f}\right)$ mapping $U$ to $Y$ is of rank at most $N$ and can be factorized as follows :

$$
\mathcal{H}\left(k, k_{i}, k_{f}\right)=\mathcal{O}\left(k, k_{f}\right) \mathcal{C}\left(k, k_{i}\right) .
$$

The submatrices of the factors satisfy the following recurrences :

$$
\begin{aligned}
\mathcal{O}_{j} & =\left[\begin{array}{c}
C_{j} \\
\mathcal{O}_{j+1} A_{j}
\end{array}\right], j=k_{f}-1, \ldots, k, \mathcal{O}_{k_{f}}=C_{k_{f}}, \\
\mathcal{C}_{j+1} & =\left[\begin{array}{cc}
B_{j} & A_{j} \mathcal{C}_{j}
\end{array}\right], j=k_{i}+1, \ldots, k-1, \mathcal{C}_{k_{i}+1}=B_{k_{i}} .
\end{aligned}
$$

These recurrences construct the controllability matrix forward from $k_{i}$ to $k-1$ and the observability matrix backward from $k_{f}$ to $k$. The idea of our Recursive Low-Rank Hankel approximation method is now to compute these recurrences using low rank approximations at each time step, according to the following recursive scheme :

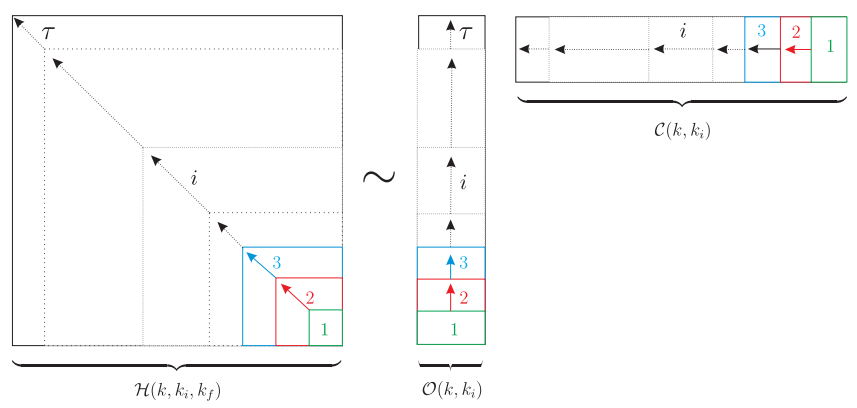


Algorithm 1. Let the $N \times n$ initializing matrices $S_{c}\left(k_{i}\right)=B_{k_{i}}$ and $S_{o}\left(k_{f}\right)=C_{k_{f}}$, then the $i^{\text {th }}$ $(i=1, \ldots, \tau)$ low-rank approximations $S_{c}\left(k_{i}+i\right)$ and $S_{o}\left(k_{f}-i\right)$ are obtained as follows :

$$
\begin{aligned}
{\left[S_{c}\left(k_{i}+i\right) \mid E_{c}\left(k_{i}+i\right)\right] } & =(I)\left[V^{(1)}(i) \mid V^{(2)}(i)\right](4) \\
{\left[\frac{S_{o}^{T}\left(k_{f}-i\right)}{E_{o}^{T}\left(k_{f}-i\right)}\right] } & =\left[\frac{U^{(1) T}(i)}{U^{(2) T}(i)}\right](I I)
\end{aligned}
$$

where $(I),(I I)$ denote :

$$
\begin{gathered}
(I)=\left[B_{k_{i}+i-1} \mid A_{k_{i}+i-1} S_{c}\left(k_{i}+i-1\right)\right], \\
(I I)=\left[\frac{C_{k_{f}-i}}{S_{o}^{T}\left(k_{f}-i+1\right) A_{k_{f}-i}}\right]
\end{gathered}
$$

$U^{(1)}(i) \in \mathbb{R}^{(p+n) \times n}$ and $V^{(1)}(i) \in \mathbb{R}^{(m+n) \times n}$ come from the $S V D$ of the product $(I I) .(I)$ :

$$
(I I) .(I)=\underbrace{U_{h}(i) \Sigma(i) V_{h}(i)^{T}}_{S V D}
$$

Let us investigate the amount of work involved in our algorithm: first we need to form products of the type $A_{j} S_{c}(j)$ and $S_{o}^{T}(l+1) A_{l}$. If we assume the matrices $A_{k}$ to be sparse ${ }^{3}$, then the amount of work needed for this is $\Theta(\alpha N n)$ [5]. The construction of the left hand side of $(6)$ requires an additional $2 N(n+m)(n+p)$ flops and the application of the transformations $U$ and $V$ requires $\Theta((p+n)(m+n)(2 n+p+m))$ flops, and so the complexity of this algorithm is $\Theta(N(p+$ $n)(m+n))$ for each iteration.

Now in order to make the link between the whole controllability and observability matrices $\mathcal{C}(.,),. \mathcal{O}(.,$. and their low-rank approximations $S_{c}($.$) and S_{o}($.$) ,$ we have the following theorem :

Theorem 1. ${ }^{4}$ At each iteration, there exist orthogonal matrices $V_{i} \in \mathbb{R}^{(n+i m) \times(n+i m)} \quad$ and $U_{i} \in \mathbb{R}^{(n+i p) \times(n+i p)}$ satisfying : $\left(l=k_{i}+i, \quad r=\right.$ $\left.k_{f}-i\right)$

$$
\begin{aligned}
\mathcal{C}\left(l, k_{i}\right) V_{i} & =\left[S_{c}(l)\left|E_{c}(l)\right| \ldots \mid A_{l-1} \mathcal{C}_{e}\left(l, k_{i}\right)\right], \\
\mathcal{O}^{T}\left(r, k_{f}\right) U_{i} & =\left[S_{o}(r)\left|E_{o}(r)\right| A_{r}^{T} \mathcal{O}_{e}^{T}\left(r+1, k_{f}\right)\right],
\end{aligned}
$$
where $E_{c}\left(k_{i}+i\right)$ and $E_{o}\left(k_{f}-i\right)$ are the neglected parts at iteration $i$ (4) and

$$
\begin{aligned}
& \mathcal{C}_{e}\left(l, k_{i}\right) \doteq\left[E_{c}(l-1)|\ldots| \Phi\left(l-1, k_{i}\right) E_{c}\left(k_{i}\right)\right] \\
& \mathcal{O}_{e}\left(r, k_{f}\right)^{T} \doteq\left[E_{o}(r)|\ldots| \Phi\left(k_{f}, r\right)^{T} E_{o}\left(k_{f}\right)\right] .
\end{aligned}
$$

\footnotetext{
${ }^{3} \alpha$ the number of non-zero elements per row or column of $A_{k}$.

${ }^{4}$ The proof is very similar to that of Theorem.1 of [2], and is left out here for sake of brevity.
}

As a consequence of this theorem we have the following result which give us an approximation of the original Hankel map $\mathcal{H}\left(k, k_{i}, k_{f}\right)$ :

Corollary 2. There exist orthogonal matrices $V_{k} \in \mathbb{R}^{(n+\tau m) \times(n+\tau m)}$ and $U_{k} \in \mathbb{R}^{(n+\tau p) \times(n+\tau p)}$ such that :

$$
\begin{aligned}
& U_{k}^{T} \mathcal{H}\left(k, k_{i}, k_{f}\right) V_{k}= \\
& {\left[\begin{array}{ccc}
S_{o}^{T}(k) S_{c}(k) & 0 & S_{o}^{T}(k) A_{k-1}(I I I) \\
0 & E_{o}^{T}(k) E_{c}(k) & E_{o}^{T}(k) A_{k-1}(I I I) \\
(I V) A_{k} S_{c}(k) & (I V) A_{k} E_{c}(k) & (I V) A_{k} A_{k-1}(I I I)
\end{array}\right],}
\end{aligned}
$$

where

$$
(I I I)=\mathcal{C}_{e}\left(k, k_{i}\right), \quad \text { and } \quad(I V)=\mathcal{O}_{e}\left(k+1, k_{f}\right) .
$$

This result will enable us to evaluate the quality of our approximations by using the Hankel operator without having to pass via Gramians, which can be very useful in some cases.

\section{Model reduction of LTI system}

In this section we analyze the quality of our approximation for the time-invariant case. In this special case we run the above procedure on the interval $[-\tau, \tau]^{5}$, and we obtain two matrices $S_{c}$ and $S_{o}$ of full rank $n$. First, using those matrices we can approximate the Gramians of the original model $\mathcal{S}$ by $\mathcal{P}_{\tau}=S_{c} S_{c}^{T}$ and $\mathcal{Q}_{\tau}=S_{o} S_{o}^{T}$. The difference between the approximate low-rank Gramians and the exact Gramians

$$
\mathcal{E}_{c}(\tau) \doteq \mathcal{G}_{c}(\tau)-\mathcal{P}_{\tau}, \quad \mathcal{E}_{o}(\tau) \doteq \mathcal{G}_{o}(\tau)-\mathcal{Q}_{\tau}
$$

remains bounded for large $\tau$, as indicated in the following theorem.

Theorem 3. ${ }^{6}$ Let $\mathcal{P}$ and $\mathcal{Q}$ be respectively the solutions of $\mathcal{P}=A \mathcal{P} A^{T}+I$ and $\mathcal{Q}=A^{T} \mathcal{Q} A+I$, then

$$
\left\|\mathcal{E}_{c}(\tau)\right\|_{2} \leq \eta_{c}^{2}\|\mathcal{P}\|_{2} \leq \eta_{c}^{2} \frac{\kappa(A)^{2}}{1-\rho(A)^{2}},
$$

and

$$
\begin{gathered}
\left\|\mathcal{E}_{o}(\tau)\right\|_{2} \leq \eta_{o}^{2}\|\mathcal{Q}\|_{2} \leq \eta_{o}^{2} \frac{\kappa(A)^{2}}{1-\rho(A)^{2}} \\
\text { where } \eta_{c} \doteq \max _{\tau}\left\|E_{c}(\tau)\right\|_{2} \text { and } \eta_{o} \doteq \max _{\tau}\left\|E_{o}(\tau)\right\|_{2} .
\end{gathered}
$$

\footnotetext{
${ }^{5}$ Which means that we choose $k_{i}=-\tau$ and $k_{f}=\tau(\tau \in \mathbb{N})$.

${ }^{6}$ The proof is very similar to that of Theorem.2 of [2], and is left out here for sake of brevity.
} 
Theorem 4. Using the first $n$ columns $U_{\tau}^{(1)}$ of $U_{\tau}$ and $V_{\tau}^{(1)}$ of $V_{\tau}$, we obtain a rank $n$ approximation of the Hankel map :

$$
\mathcal{H}(\tau)-U_{\tau}^{(1)} S_{o}^{T}(\tau) \cdot S_{c}(\tau) V_{\tau}^{(1) T}=\mathcal{E}_{h}(\tau),
$$

for which we have the error bound :

$$
\begin{gathered}
\left\|\mathcal{E}_{h}(\tau)\right\|_{2} \leq \\
\frac{\kappa(A)}{\sqrt{1-\rho(A)^{2}}} \max \left\{\eta_{c}\left\|S_{o}^{T} A\right\|_{2}, \eta_{o}\left\|A S_{c}\right\|_{2}\right\}+\frac{\kappa(A)^{2}}{1-\rho(A)^{2}} \eta_{o} \eta_{c} .
\end{gathered}
$$

Proof. This follows directly from the bounds of Theorem. 3 that can be used to bound the blocks in the form in $(7)$ different from the $(1,1)$ block.

Remark 1. In the time-invariant case all matrices $A, B$ and $C$ are constant. As a consequence all Hankel maps of width $\tau$ are equal as well and only the interval width plays a role in the obtained decomposition. As a consequence one obtains an approximate rank factorization of a Hankel map with $i$ block columns and rows at each instant $i$. The bounds obtained in Theorem.3 and 4 are moreover independent of $\tau$. As $i$ grows larger one can expect that reasonable approximations of $\eta_{c}$ and $\eta_{o}$ are in fact given by the last terms, i.e. $\eta_{c} \approx\left\|E_{c}(\tau)\right\|_{2}$ and $\eta_{o} \approx\left\|E_{o}(\tau)\right\|_{2}$ which will give much tighter bounds in these theorems.

\section{Numerical example}

In this section we apply our algorithm to the International Space Station model (see [1] for a brief description of this model). The order of this system is $N=270$, it has 3 inputs and 3 outputs $(m=p=3)$, the order of the reduced system is $n=32$. We run our algorithm with $\tau=3 N$. We show the $\mathcal{H}_{\infty}$ norms of the original system and of the error systems obtained using the BT method and the RLRH method. In the figure, the $\sigma_{\max }$-plot of the full order and the corresponding error system are shown. It can be seen from the figure and the table that the recursive lowrank Hankel approximation yields results which are close to those obtained using Balanced Truncation.

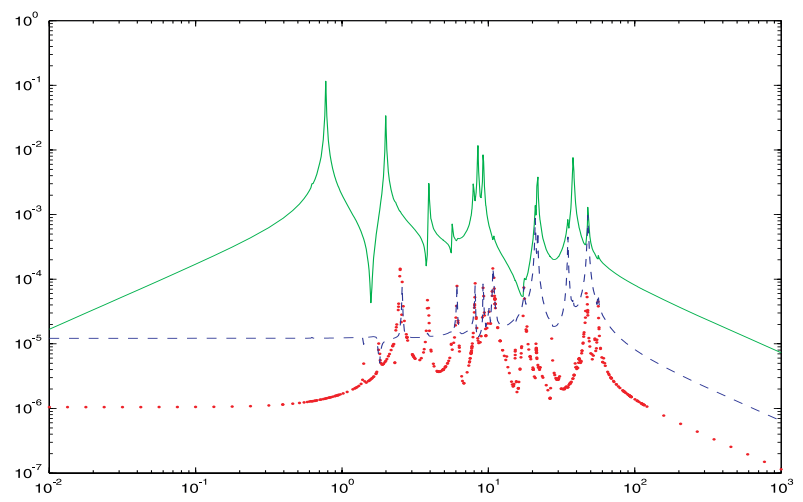

Frequency response - full model,

... BT error system, -- ILRH error system.

\begin{tabular}{|c|c|c|}
\hline$\|\mathcal{S}\|_{\mathcal{H}_{\infty}}$ & $\left\|\mathcal{S}-\mathcal{S}_{B T}\right\|_{\mathcal{H}_{\infty}}$ & $\|\mathcal{S}-\hat{\mathcal{S}}\|_{\mathcal{H}_{\infty}}$ \\
\hline 0.1159 & $2.3630 \mathrm{e}-004$ & 0.0011 \\
\hline
\end{tabular}

\section{Conclusions}

In this paper we propose a new model reduction method based on a recursive low rank approximation of the time-varying Hankel map. We applied this to both the time-varying and time-invariant case and showed error bounds for the approximation of the Gramians and Hankel map. Numerical experiments show that our approach provides a comparable results to those obtained by Balanced Truncation but with a significantly reduced computational cost. Although this Hankel map factorization approach has obvious advantages, many open questions remain regarding performance, convergence, and accuracy which require further analysis.

\section{References}

[1] Y. Chahlaoui, P. Van Dooren. "A collection of benchmarks examples for model reduction of linear time invariant dynamical systems", SLICOT Working note 2002-2, via http://www.win.tue.nl/niconet/niconet.html, (2002)

[2] Y. Chahlaoui, P. Van Dooren. "Estimating Gramians of large-scale time-varying systems", Proceedings of the 15th Triennial World Congress, IFAC02, Barcelona, Spain, (2002).

[3] D. F. Enns. "Model reduction with balanced realizations: An error bound and frequency weighted generalization", Proc. of the IEEE Conference on Decision and Control, pp. 127-132, (1984).

[4] K. Glover. "All optimal Hankel norm approximations of linear multivariable systems and their $\mathcal{L}^{\infty}$ - 
error bounds", Internat. J. Control, 39, pp. 11151193, (1984).

[5] G. Golub, C. Van Loan. "Matrix computations", The Johns Hopkins University Press, (1996).

[6] E. Verriest, T. Kailath. "On generalized balanced realizations", IEEE Trans. Automat. Contr., AC28, pp. 833-844, (1983).

[7] B. C. Moore. "Principal component analysis in linear systems: controllability, observability, and model reduction", IEEE Trans. Automat. Control, 26, pp. 17-31, (1981).

[8] H. Sandberg, H. Rantzer. "Balanced model reduction of linear time-varying systems", IFAC02, 15th Triennial World Congress, Barcelona, Spain, (2002).

[9] S. Shokoohi, L. M. Silverman, P. M. Van Dooren. "Linear time-variable systems: Balancing and model reduction", IEEE Trans. Automat. Control, 28, pp. 810-822, (1983).

[10] P. Van Dooren. "Gramian based model reduction of large-scale dynamical systems", in Numerical Analysis 1999, pp. 231-247, (2000).

[11] M. Verlaan, A. Heemink. "Tidal flow forecasting using reduced rank square root filters", Stochastic Hydrology and Hydraulics, 11, pp. 349-368, (1997).

[12] P. Dewilde, A. van der Veen. "Time-varying systems and computations", Kluwer Academic Publishers, (1998). 